\title{
Fabry disease - what cardiologists can learn from the nephrologist: a narrative review
}

\author{
Christine E. Kurschat ${ }^{1,2,3 \wedge}$ \\ ${ }^{1}$ Department II of Internal Medicine and Center for Rare Diseases Cologne, University Hospital of Cologne, Cologne, Germany; ${ }^{2}$ Cologne \\ Excellence Cluster on Cellular Stress Responses in Ageing-Associated Diseases (CECAD), University of Cologne, Cologne, Germany; ${ }^{3}$ Center for \\ Molecular Medicine Cologne, University of Cologne, Cologne, Germany \\ Correspondence to: Christine Kurschat, MD. Department II of Internal Medicine, Nephrology, Rheumatology, Diabetes and General Medicine, \\ University Hospital of Cologne, Kerpenerstr. 62, 50937 Cologne, Germany. Email: christine.kurschat@uk-koeln.de.
}

\begin{abstract}
Fabry disease (FD) is a rare, X-linked lysosomal storage disorder resulting in decreased or absent activity of the lysosomal enzyme alpha-galactosidase A. Subsequent accumulation of storage material can occur in virtually all cells of the body. Organs and structures affected by storage material deposition include the heart, the kidney, the central and peripheral nervous system and the cornea of the eyes. Progressive cardiac hypertrophy, arrhythmias, cardiac fibrosis, heart failure and cardiac death are common characteristics of cardiac involvement. Renal depositions of glycosphingolipids are already detectable in childhood. An early clinical sign of Fabry renal involvement is albuminuria, often preceding a detectable loss of kidney function. Later in life Fabry patients may exhibit a progressive decline of their kidney function leading to end-stage renal disease (ESRD). The clinical presentation of Fabry patients regarding renal involvement depends on the underlying mutation in the GLA gene. Classically affected males typically show a gradual decrease in kidney function, patients with mild or late onset mutations as well as a subgroup of females may exhibit only little or no renal abnormalities. This review summarizes the characteristics of renal involvement in FD, the diagnostics necessary to evaluate the degree of renal impairment and possible treatment options.
\end{abstract}

Keywords: Fabry disease (FD); proteinuria, albuminuria; renal insufficiency, end-stage renal disease (ESRD)

Submitted Dec 04, 2020. Accepted for publication Mar 10, 2021.

doi: $10.21037 / \mathrm{cdt}-20-981$

View this article at: http://dx.doi.org/10.21037/cdt-20-981

\section{Introduction}

Fabry disease (FD, OMIM \# 301500) is a lysosomal storage disorder due to mutations in the GLA gene on the $\mathrm{X}$ chromosome, encoding the lysosomal enzyme alphagalactosidase A (1). These mutations lead to reduced or abrogated enzyme activity. To date, more than 1,000 mutations in the GLA gene have been identified. In the lysosome, alpha galactosidase A cleaves the terminal galactose molecule from globotriaosylceramide (Gb3). Reduced enzyme levels lead to accumulation of $\mathrm{Gb} 3$ and its deacylated form globotriaosylsphingosine (lyso-Gb3) in virtually all cells of the body. Usually, FD is more severe in males, whereas in females the disease burden largely depends on the $\mathrm{X}$ chromosome inactivation pattern. Organs predominantly affected in FD are the kidneys, the heart and the central and peripheral nervous system. Although accumulation of storage material already starts pre-birth, symptoms of FD usually develop in early or late childhood (2). Children exhibit burning sensations in hand and feet as a sign of neuropathic pain. Hypohidrosis, recurrent fever episodes, pain crises, and angiokeratoma may also be present. Corneal sphingolipid depositions are detectable by slit lamp examination, called cornea verticillata. With increasing age organ dysfunction

\footnotetext{
^ ORCID: 0000-0002-3646-9471.
} 
becomes more and more apparent. Fabry patients develop proteinuria, albuminuria and decreasing kidney function which may progress to end-stage renal disease (ESRD) with need for dialysis or renal transplantation. Heart involvement is diverse, ranging from increasing left ventricular hypertrophy, mostly without hypertension, bradycardia, rhythm abnormalities and fibrosis, to cardiac insufficiency, the need for heart transplantation, and sudden cardiac death. Recurrent stroke episodes are a hallmark for central nervous involvement, often debilitating and decreasing quality of life. Due to major advances in treating renal involvement, cardiovascular disease has become the major cause of death in patients with FD (3).

For establishing the diagnosis of FD in males, enzymatic analysis from dried blood spots is used to determine low alpha-galactosidase A enzyme activity. In most males enzyme activity is reduced to less than $30 \%$ of the normal range with no activity present in some individuals. Diagnosis is confirmed by genetic testing revealing the underlying mutation. In heterozygous females genetic testing is mandatory to establish the diagnosis since alphagalactosidase A activity in leucocytes may be normal despite low levels in other organs.

Since 2001, enzyme replacement therapy (ERT) is available: agalsidase alfa $\left(\right.$ Replagal $^{\circledR}$, Takeda, dose $0.2 \mathrm{mg} / \mathrm{kg}$ body weight every 2 weeks) and agalsidase beta (Fabrazyme ${ }^{\circledR}$, Sanofi-Genzyme, dose $1.0 \mathrm{mg} / \mathrm{kg}$ body weight every 2 weeks). Recently, the first oral therapy became available: migalastat (Galafold ${ }^{\circledR}$, Amicus Therapeutics, $123 \mathrm{mg}$ every other day) $(4,5)$. While ERT aims at replacing the defective enzyme by infusion of recombinant protein, migalastat is a chaperone enhancing residual patient enzyme activity. Thus, migalastat is only indicated in patients with mutations responsive to migalastat treatment (amenable mutations). Amenability is tested by a standardized in vitro assay and should be verified for the specific patient mutation before initiation of treatment (https://www. galafoldamenabilitytable.com). Thirty-month results on the long-term efficacy and safety of migalastat have recently been published demonstrating stable kidney function and sustained reduction in left ventricular mass (ATTRACT study) (6).

The search for specific biomarkers in FD is ongoing (7). To date, Gb3 and lyso-Gb3 in dried blood spots, plasma, serum and urine can be measured by mass spectrometry $(8,9)$. Lyso-Gb3 levels, although not ideal, are thought to be superior in estimating the burden of disease and in monitoring disease progression as well as effectiveness of
Fabry therapy (9).

Fabry patients should be monitored regularly once every 6 to 12 months, with special interest in renal, heart and nervous system alterations. In this review, we focus on evaluation of renal involvement by the nephrologist. We present the following article in accordance with the Narrative Review reporting checklist (available at http:// dx.doi.org/10.21037/cdt-20-981).

\section{Renal involvement in FD}

\section{Clinical presentation}

In most patients, proteinuria and albuminuria are the earliest clinical signs of Fabry renal involvement in males and females $(10,11)$. The degree of proteinuria is an independent risk factor for the extent of declining renal function over time (12). Patients with more severe proteinuria progress more rapidly to ESRD compared to patients with only mild proteinuria (13). Thus, treatment of Fabry renal disease should aim at reducing proteinuria. Measuring albuminuria instead of proteinuria is even more precise in establishing renal involvement of FD. Albumin is only detectable in the urine if the renal glomerular filter, consisting of glomerular podocytes, endothelial cells and the glomerular basement membrane, is damaged. In contrast, proteinuria can also occur without renal damage, e.g., with urologic diseases, and be falsely measured in the urine with gynecological disease. The prevalence of proteinuria in Fabry patients ranges between $44-54 \%$ in hemizygous males and $33-41 \%$ in heterozygous females (14). Hematuria may also be detectable in Fabry patients although this finding is rather uncommon $(12,15)$. If hematuria is present in a patient with FD other causes of hematuria must be excluded. Blood pressure is usually low in Fabry patients with only mild or no kidney involvement, although they may present with left ventricular hypertrophy due to storage material. With progression of kidney disease to higher stages, blood pressure increases and hypertension may develop (16). In these patients, treatment of hypertension is important because elevated blood pressure levels contribute to further decline of kidney function.

Proteinuria in the nephrotic range $(>3.5 \mathrm{~g} /$ day $)$ is rather uncommon in FD. In these patients renal biopsy is recommended in order to exclude additional underlying renal pathology independent of FD.

Using renal ultrasound, kidney cysts, mostly parapelvic cysts, can be detected in up to $50 \%$ of patients $(17,18)$. The etiology of these parapelvic cysts is not known. 

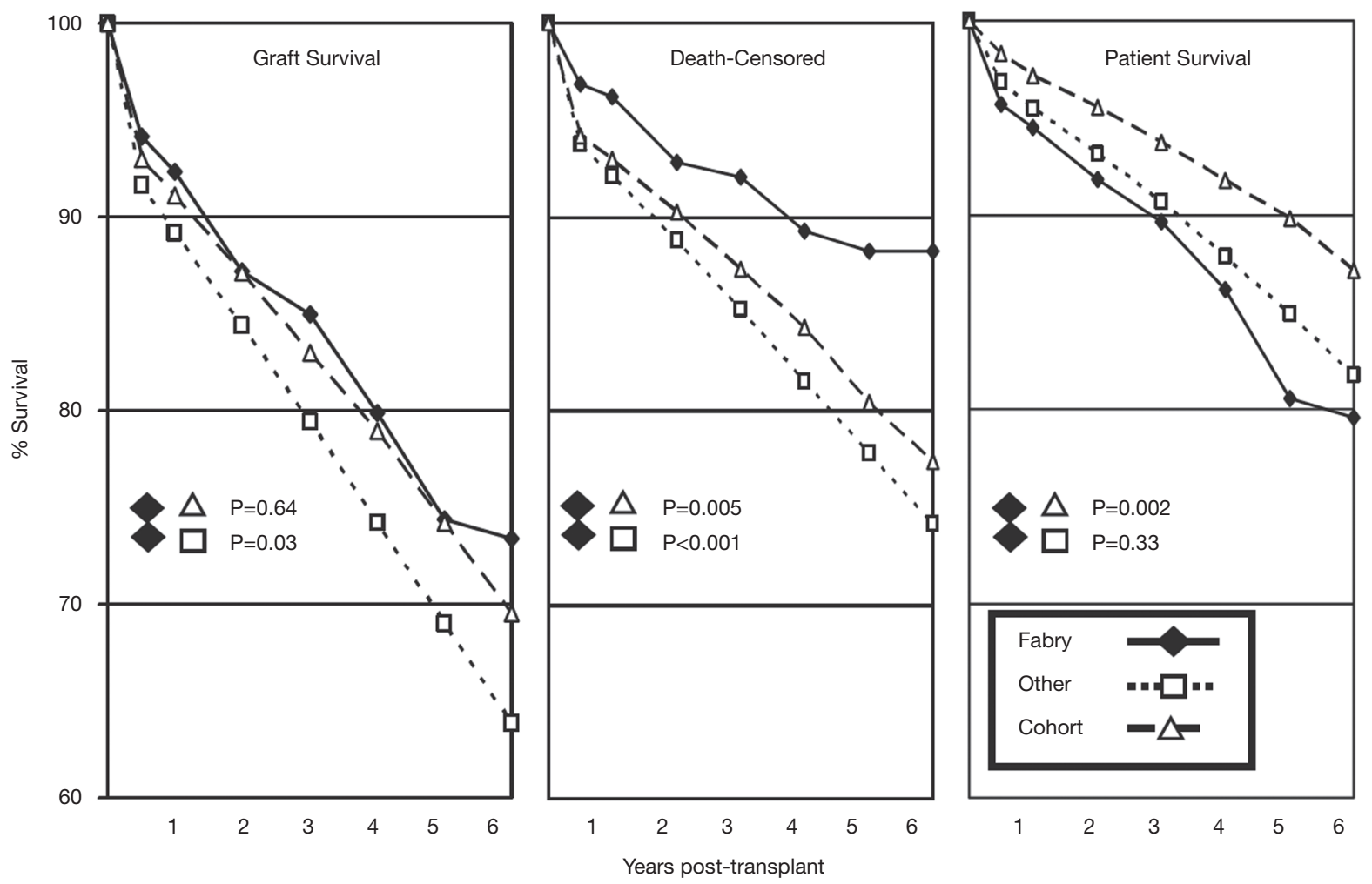

Figure 1 Graft survival, dialysis-free (death-censored) survival, and patient survival for patients with Fabry (n=197), other causes of endstage renal disease (other) and a 10:1 matched cohort of transplant recipients with other causes of ESRD (26). ESRD, end-stage renal disease.

Urinalysis may reveal typical birefringent lipid globules representing tubular cells full of storage material.

Renal involvement in female Fabry patients is also clinically detectable although it may be less overt and less severe compared to males. In a Fabry database adult females $(\mathrm{n}=638)$ had an estimated glomerular filtration rate (eGFR) $<90 \mathrm{~mL} / \mathrm{min} / 1.73 \mathrm{~m}^{2}$ in $62.5 \%$ of cases and an eGFR $<60 \mathrm{~mL} / \mathrm{min} / 1.73 \mathrm{~m}^{2}$ in $19.0 \%$ of cases (10). Proteinuria was detected in $30-35 \%$ of females, $13 \%$ of females had chronic kidney disease stage 3 and $1-4 \%$ exhibited ESRD (12).

Renal FD may progress to ESRD in untreated males by the third to fifth decade, with age ranging from as early as 16 years to 63 years $(19,20)$. The degree of renal function decline varies in affected males and females, even within the same family exhibiting the same underlying GLA mutation. It has been shown that the severity of alpha-galactosidase A deficiency contributes, at least in part, to the rate of progression (20). However, additional factors such as modifier genes or environmental influences may be involved in the degree of progression to ESRD. The reason for progression to renal failure is not clear. Glycosphingolipid deposits are already detectable at a very young age in children despite normal renal function $(11,21)$. Declining renal function may be determined by the severity of intrarenal vascular damage as a consequence of storage material deposition in endothelial cells of the renal vasculature eventually leading to ischemic nephropathy (19).

In ESRD, dialysis and renal transplantation are the options of treatment. Several publications revealed that Fabry patients on dialysis exhibit a reduced life-span compared to dialysis patients with other underlying diseases (22). Thus, kidney transplantation should be the first choice in Fabry patients (23). Renal transplantation prolongs life-span of Fabry patients compared to Fabry dialysis patients and yields excellent results $(24,25)$. However, life-span has been shown to be reduced in Fabry transplant patients compared to transplant patients having received a kidney due to alternative reasons (Figure 1) (26).

Fabry-specific depositions usually do not recur in 


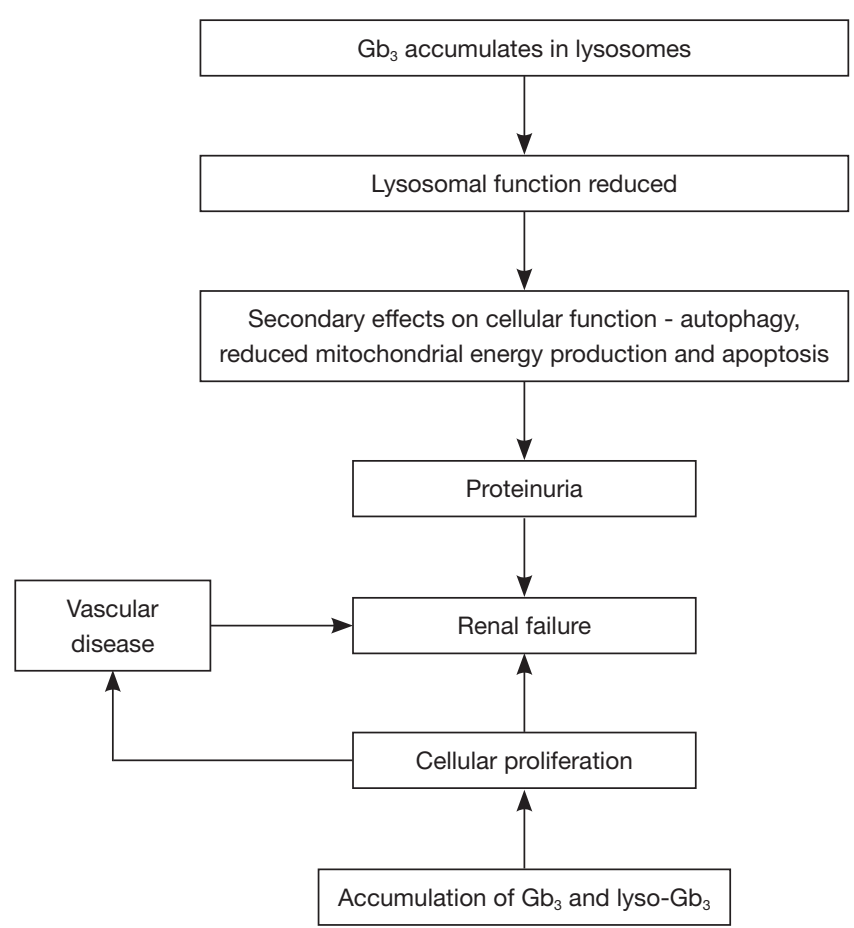

Figure 2 Pathogenesis of Fabry nephropathy. Gb3, globotriaosylceramide; lyso-Gb3, globotriaosylsphingosine (12).

the transplant since the transplant kidney expresses normal levels of alpha-galactosidase A. Occasionally, glycosphingolipid material was detected in the transplant, mainly in non-resident immune cells and endothelial cells $(25,27,28)$. In the era before ERT was available severe deposition of storage material was reported 14 years after transplantation (29). In the context of living kidney donation several case reports discuss accidental kidney donation of Fabry patients, mostly females $(19,30)$. Thorough work-up of the kidney donor is mandatory if renal abnormalities are detectable, including a renal biopsy. Even with an extensive clinical work-up FD in female donors may be missed since renal involvement is absent in a substantial subgroup of female patients.

Fabry-specific therapy should be continued after renal transplantation since FD is a multi-systemic disorder not only affecting the kidney but many other organs. It is safe to maintain Fabry dialysis and renal transplant patients on ERT with the same dosing regimen.

\section{Histopathology}

The pathology of Fabry renal disease is not fully understood (12). Initial deposition of Gb3 in renal cellular lysosomes leads to impaired lysosomal function, intracellular changes and histopathological damage (Figure 2).

In advanced Fabry nephropathy histological alterations are already detectable on light microscopy $(31,32)$ (Figure 3). Glomeruli show prominent vacuolization of podocytes, mesangial cells and endothelial cells, reflecting inclusion of storage material, mainly Gb3. Storage material is also present in distal tubular cells, cells of the loop of Henle and collecting duct, and, to a lesser extent, in parietal epithelial cells. Depositions in proximal tubular cells are uncommon (33). Hyaline deposits are found in the media of renal arteries and arterioles. During routine paraffine histology processing cytoplasmic Gb3 depositions are removed. With disease progression podocyte foot process effacement becomes apparent, a hallmark for increasing proteinuria (34-36). Endothelial fenestration decreases and duplication of the glomerular basement membrane is detectable. These alterations lead to inflammatory reactions resulting in thickening of vascular walls, focal segmental and global glomerulosclerosis, interstitial fibrosis and tubular atrophy. This is a common final path and a "point of no return" for the development of ESRD also present in other renal pathologies.

An adequate method to demonstrate Fabry-specific deposits on light microscopy is toluidine blue staining (Figure 3F,G) $(31,32)$. This method is not part of routine pathological workup and has to be requested if Fabry nephropathy is a differential diagnosis. This staining yields dark blue cytoplasmic inclusions in glomerular podocytes, tubular cells and renal arterioles. Fogo et al. developed a Fabry renal histology classification system to determine prognosis and to quantify treatment response (33). Specific characteristics of Fabry nephropathy are readily detectable on electron microscopy showing intracellular lamellated membrane structures called zebra bodies or myelin bodies (Figure $3 A, B, C$ ). These findings are not only pathognomonic for FD but are also detectable upon hydroxychloroquine treatment $(31,37)$. For diagnosing FD renal histologic evidence is usually not required if clinical, biochemical and genetic diagnosis is well established. In some patients FD is diagnosed by chance in a renal biopsy.

\section{Renal involvement in children}

In children the first clinical presentation of FD is mainly neuropathic pain with burning sensations in hands and feet. In the early stages of the disease gastrointestinal symptoms, 

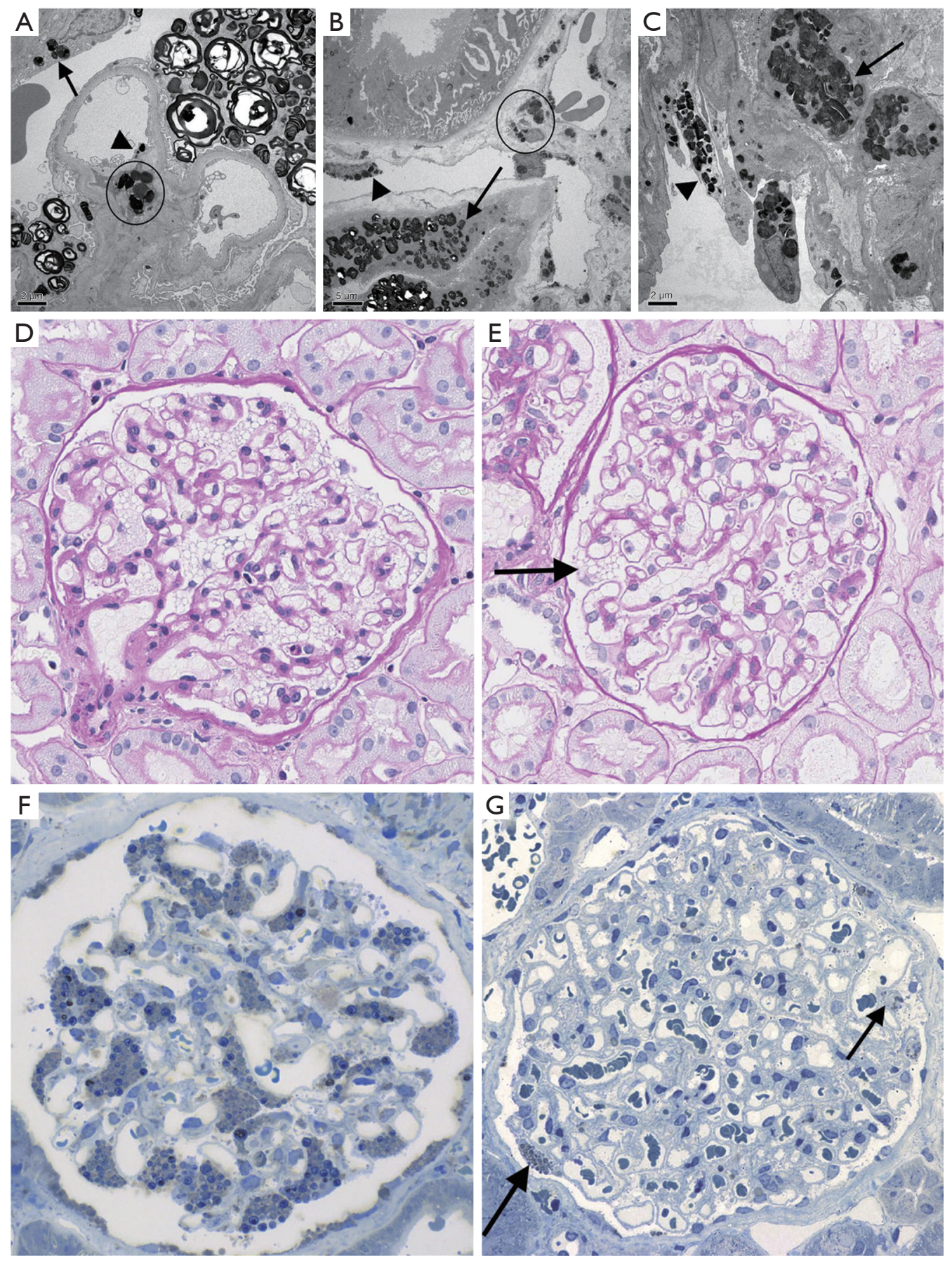

Figure 3 Kidney biopsy electron microscopic images of GL-3 inclusions (A,B,C). (A) Glomerulus with inclusions in podocytes, mesangium cells (circle), endothelial cells (arrowhead) and parietal epithelial cells (arrow). (B) Inclusions in distal tubules (arrow), endothelial cells of peritubular capillaries (arrowhead) and interstitial cells (circle). Proximal tubules are spared. (C) Arteriole with inclusions in endothelial cells (arrowhead) and smooth muscle cells (arrow). Light microscopy of glomeruli with Fabry disease (D,E,F,G). GL-3 deposits are dissolved when biopsy tissue is processed and embedded in paraffin. Periodic acid Schiff-stained sections (D,E) from paraffin-embedded renal biopsies. (D) Vacuolization of all podocytes. (E) Only one podocyte shows some vacuoles (arrow). GL-3 deposits are preserved when embedded in semi-thin plastic-embedded toluidine blue-stained sections (F,G). (F) Most podocytes are expanded by GL-3 deposits. (G) Podocytes show rare inconspicuous deposits (arrows). Modified from reference (32). 
Table 1 Definition of chronic kidney disease (CKD) stages by glomerular filtration rate (GFR) and albuminuria (A)

\begin{tabular}{lcc}
\hline CKD stage & Level & Description \\
\hline G1 & GFR $>90 \mathrm{~mL} / \mathrm{min} / 1.73 \mathrm{~m}^{2}$ & With other evidence of chronic kidney damage \\
G2 & GFR $60-89 \mathrm{~mL} / \mathrm{min} / 1.73 \mathrm{~m}^{2}$ & Mild impairment \\
G3a & GFR $45-59 \mathrm{~mL} / \mathrm{min} / 1.73 \mathrm{~m}^{2}$ & Moderate impairment \\
G3b & GFR $30-44 \mathrm{~mL} / \mathrm{min} / 1.73 \mathrm{~m}^{2}$ & Severe impairment \\
G4 & GFR $15-29 \mathrm{~mL} / \mathrm{min} / 1.73 \mathrm{~m}^{2}$ & Established renal failure \\
G5 & GFR $<15 \mathrm{~mL} / \mathrm{min} / 1.73 \mathrm{~m}{ }^{2}$ or on dialysis & \\
A1 & Albuminuria $<30 \mathrm{mg} / \mathrm{g}$ creatinine $(\mathrm{ACR}$, albumin-to-creatinine ratio) & \\
A2 & Microalbuminuria $30-300 \mathrm{mg} / \mathrm{g}$ creatinine & \\
A3 & Albuminuria $>300 \mathrm{mg} / \mathrm{g}$ creatinine & \\
\hline
\end{tabular}

hypohidrosis, and fever are also common. Clinical signs are reported as early as 3 years in boys and 6 years in girls with a mean age at presentation of 10 years in boys and 15 years in girls. Fabry nephropathy remains clinically silent for many years. Histologic evidence of deposition of storage material is already detectable at a very young age and precedes development of albuminuria $(11,21)$. Albuminuria may be present below 10 years of age although, in most patients, renal involvement becomes clinically apparent later in life $(11,21)$. Tøndel et al. identified the combination of neuropathic pain, mild albuminuria, glomerular endothelial cell deposits, and arteriopathy in children to present a risk factor for potentially progressive kidney disease (11). In a cohort of 14 children (age ranging from 4 to 19 years) sphingolipid inclusion volume of podocytes was shown to increase with age (35). Progression to ESRD in children is uncommon although it was reported as early as 16 years of age (38).

Early diagnosis of Fabry nephropathy is crucial since early initiation of therapy may halt progressive renal dysfunction more effectively compared to late initiation. By the time kidney disease becomes clinically evident, irreversible lesions in the kidney may already have developed. In the absence of sensitive early, noninvasive biomarkers of renal involvement in children and adults, establishing the diagnosis of Fabry nephropathy is challenging. It has been demonstrated that glycospingolipid deposition in the kidney, to date only detectable by renal biopsy, precedes clinical evidence of renal involvement. Thus, providing evidence for early Fabry renal involvement in children may only be possible by renal biopsy. In the future, testing urinary cells including podocytes of children with FD might be a less invasive option $(39,40)$. To date, techniques to assess pathology in urinary podocytes are not standardized and only available in research laboratories. Timing of initiation of treatment is important considering the burden of intravenous administration of ERT and the high costs of therapy.

\section{How to evaluate renal involvement in FD}

To date, proteinuria, albuminuria and progressive reduction of glomerular filtration rate (GFR) are the only established markers of renal dysfunction in Fabry patients. These parameters are necessary to evaluate renal disease and to monitor disease progression.

\section{Renal function}

Renal function is usually assessed by repeated measurements of serum creatinine. Creatinine values are the basis for calculating estimated glomerular filtration rate (eGFR). In adults, the formula currently used by nephrologists worldwide is the CKD-EPI (2009) formula since this equation proved to be superior to the MDRD formula used previously. In children, the Schwartz formula is currently used. Normal eGFR values should range above $90 \mathrm{~mL} / \mathrm{min} / 1.73 \mathrm{~m}^{2}$. eGFR yields a more precise picture of declining renal function, especially between 50 $90 \mathrm{~mL} / \mathrm{min} / 1.73 \mathrm{~m}^{2}$ where creatine values may still be within the normal range. Today, the degree of renal impairment, regardless of the underlying disease, is categorized according to GFR values, combined with the level of albuminuria (KDIGO 2012, Table 1). 


\section{Proteinuria}

Proteinuria is a frequent indicator of Fabry renal involvement, present in $44-54 \%$ of males and $33-41 \%$ of females $(14,41)$. Measuring albuminuria is even more precise since albuminuria is detectable only in the presence of glomerular podocyte damage. Proteinuria is an independent risk factor for the extent of renal involvement. It is a valuable tool in assessing the degree of renal disease, monitoring renal function with time and determining the effects of Fabry treatments.

Proteinuria as well as albuminuria should be monitored regularly and treated appropriately, if present.

\section{Additional urine assessments}

Urine microscopy reveals vacuolated epithelial cells filled with storage material, yielding the appearance of a Maltese cross using polarized light microscopy (42). Mulberry cells may also be observed in the urinary sediment being strongly suggestive of FD. These cells are believed to represent tubular cells filled with sphingolipids (43). In specialized laboratories, podocyte excretion can be assessed, which is increased in Fabry patients and decreases with Fabry therapy $(35,44)$. Urine diagnostics, apart from measurements of proteinuria and albuminuria, are usually not included in routine diagnostics and follow-up.

\section{Gb3 and Lyso-Gb3}

Gb3 and lyso-Gb3 are widely used as biomarkers in FD, determined in plasma, serum, urine and tissue. Serum levels of lyso-Gb3 are higher in male patients with classical GLA mutations compared to patients with late-onset mutations and in males with frame-shift mutations or deletions compared to missense mutations (9). Yet, prediction of clinical outcome using these molecules is still difficult. Urinary Gb3 is elevated in Fabry patients and decreases upon initiation of treatment. However, the level of urinary Gb3 does not correlate with the degree of renal damage and does not predict renal outcome (45). Lyso-Gb3 in serum is a more suitable parameter to monitor disease progression (9).

\section{Cystatin C}

Cystatin $\mathrm{C}$ is a small protease produced at a constant rate by nucleated cells. It is freely filtered by glomeruli and completely absorbed and catabolized in proximal tubular cells. The concentration of cystatin $\mathrm{C}$ in serum is sensitive to changes in GFR, especially in the early phases of renal function impairment. Cystatin $\mathrm{C}$ is a more sensitive parameter compared to creatinine to detect small decreases in GFR. Cystatin C levels do not depend on age, sex or muscle mass. In Fabry patients, cystatin $\mathrm{C}$ is a reliable marker of renal function (46).

\section{Renal ultrasound}

In up to $50 \%$ of Fabry patients renal cysts are detectable, mostly in the parapelvic region. Parapelvic renal cysts are unspecific and can also be seen in other renal diseases. The underlying pathomechanisms of Fabry parapelvic renal cysts are not known. Renal ultrasound abnormalities in FD, if present, resemble alterations also found in other chronic kidney diseases.

\section{Hypertension}

Analyzing data from international Fabry registries indicate that hypertension (blood pressure $130 / 80 \mathrm{mmHg}$ ) is detectable in up to $57 \%$ to $67 \%$ of patients $(16,41)$. Importantly, the presence of hypertension is closely related to declining renal function. In general, Fabry patients with normal renal function exhibit blood pressure levels lower than normal as a consequence of autonomic dysfunction influencing vascular and cardiac structures $(12,47)$. Low blood pressure levels complicate therapeutic intervention with ACE inhibitors or AT1 receptor antagonists in proteinuric patients.

\section{Clinical management of Fabry renal disease}

Once renal involvement of FD is established, regular monitoring of glomerular filtration rate (GFR) and proteinuria is important in order to assess disease status. The long-term goal is to slow progressive decline of renal function. General nephroprotective recommendations such as control of blood pressure levels, decreased salt intake and avoidance of nephrotoxic substances are important measures and have to be discussed with the patient. Particularly, the use of non-steroidal anti-inflammatory drugs (NSAID) such as ibuprofen may lead to acute kidney injury and should be avoided. Many Fabry patients take these agents to alleviate their neuropathic pain. In the presence of renal involvement NSAIDs should be replaced by alternative drugs such 
as paracetamol. By adhering to these recommendations slowing the gradual loss of renal function is possible. The success of these measures is largely dependent on the timing of their initiation. If they are initiated early in the course of renal disease, success rates are higher compared to late initiation when renal scarring has already occurred. The presence of Fabry-associated kidney involvement is a trigger for initiation of Fabry therapy.

As in all other kidney diseases proteinuria as well as albuminuria in Fabry patients should be treated with inhibitors of the renin-angiotensin-aldosterone system, such as ACE inhibitors or AT1 receptor blockers. These agents reduce intraglomerular pressure leading to reduced protein leakage into the urine. A combination of both drugs is not recommended. Usually, when starting ACE inhibitor or AT1 receptor blocker therapy, the dose should be slowly titrated to the maximal tolerable level. Many Fabry patients are normotensive and do not tolerate high doses of ACE inhibitors or AT1 receptor antagonists. Administering these agents at bedtime is a valuable option.

\section{Effect of ERT on kidney involvement}

Evidence for renal involvement in FD (eGFR $<80 \mathrm{~mL} / \mathrm{min}$, albuminuria $>300 \mathrm{mg} / \mathrm{g}$ creatinine) is a clear indication to start therapy. If microalbuminuria is present, ACE inhibitors or AT1 receptor antagonists are the initial treatment of choice. If renal involvement is questionable and/or microalbuminuria cannot be reduced by this intervention alone renal biopsy may be helpful to detect sphingolipid deposition (12). The therapeutic goal for initiation of therapy is stabilization of renal function. To date, ERT or chaperone therapy are available with chaperone therapy being possible for only a subset of patients depending on their underlying mutation. Is has been shown during the last decade that ERT has clear beneficial effects on Fabry kidney disease. Enzyme administration once every 2 weeks leads to a reduction of eGFR decline, thus stabilizing renal function $(13,48-51)$. It has been shown upon analysis of renal biopsies that ERT is capable of substantially clearing sphingolipid load in the kidney (21). Early initiation of therapy is more beneficial for preserving kidney function compared to initiation later in the course of the disease. In a multivariate regression analysis from the Fabry registry of 151 male and 65 female patients, adults with proteinuria $>1 \mathrm{~g} / \mathrm{g}$ creatinine exhibited further renal function decline despite ERT $(13,21)$. Male patients with only little proteinuria and patients who received ERT earlier after the onset of symptoms had stable renal function. Banikazemi et al. reported a better renal benefit if therapy was started at eGFR $>55 \mathrm{~mL} / \mathrm{min} / 1.73 \mathrm{~m}^{2}$ (52). These data suggest that early initiation of ERT is important because therapy seems to be less effective in more advanced renal disease (53). Allergic reactions and antibody formation against the infused ERT protein are observed especially in males with severely reduced or no alpha-galactosidase A activity (54). These antibodies may be of clinical relevance since they may have abilities to neutralize infused enzyme activity. A survival benefit for patients treated with ERT has been demonstrated (50).

These data, although mostly acquired within registry observations and comparisons with historic controls, are valuable in discussing ERT initiation with the newly diagnosed Fabry patient.

\section{Effect of chaperone therapy on kidney involvement}

Recently, the pharmacological chaperone migalastat was approved as first oral treatment option for FD. Resembling in structure the terminal galactose molecule of $\mathrm{Gb} 3$ it was designed to increase the patient's own enzyme activity by stabilizing misfolded protein in the endoplasmic reticulum and stimulating regular trafficking to the lysosome. Approximately $30-35 \%$ of patients are treatable with migalastat according to their underlying mutation (amenable mutation). The efficacy of migalastat was demonstrated in a six-month, placebo-controlled clinical trial in 50 adult patients with FD. In this trial, patients with migalastat treatment had a more prominent reduction in sphingolipid depositions in the kidney compared to patients on placebo (4). In general, migalastat was well-tolerated, with headache, nausea and nasopharyngitis being the main complaints. Measurements of eGFR demonstrated a stabilization of renal function $(4,5)$. Before initiation of chaperone therapy the prescribing physician has to verify the amenability of a patient's mutation to migalastat (https:// www.galafoldamenabilitytable.com).

\section{Conclusions}

Early detection of renal involvement in FD is crucial since timely therapeutic intervention has the potential of changing the course of the disease. Regular determination of serum creatinine, eGFR, cystatin $\mathrm{C}$, proteinuria and albuminuria is important to detect renal abnormalities in 
all patients, male and female. Although kidney involvement in Fabry patients can be highly variable even with the same underlying mutation, both males and females may progress to end-stage renal failure. Treatment options include ACE inhibitors or AT1 receptor antagonists to treat proteinuria and albuminuria. Early intervention with ERT or chaperone therapy may help to stabilize renal function or slow the decline to ESRD. New therapeutic options on the horizon, such as substrate reduction therapy and gene therapy, will reveal their potential to improve renal outcome.

\section{Acknowledgments}

Funding: None.

\section{Footnote}

Provenance and Peer Review: This article was commissioned by the Guest Editors (Yskert von Kodolitsch, Harald Kaemmerer, Koichiro Niwa) for the series "Current Management Aspects in Adult Congenital Heart Disease (ACHD): Part III" published in Cardiovascular Diagnosis and Therapy. The article has undergone external peer review.

Reporting Checklist: The author has completed the Narrative Review reporting checklist. Available at http://dx.doi. org/10.21037/cdt-20-981

Conflicts of Interest: The author has completed the ICMJE uniform disclosure form (available at http:// dx.doi.org/10.21037/cdt-20-981). The series "Current Management Aspects in Adult Congenital Heart Disease (ACHD): Part III" was commissioned by the editorial office without any funding or sponsorship. The author reports personal fees from Amicus Therapeutics, Chiesi, SanofiGenzyme, and Takeda, outside the submitted work and has no other conflicts of interest to declare.

Ethical Statement: The author is accountable for all aspects of the work in ensuring that questions related to the accuracy or integrity of any part of the work are appropriately investigated and resolved.

Open Access Statement: This is an Open Access article distributed in accordance with the Creative Commons Attribution-NonCommercial-NoDerivs 4.0 International License (CC BY-NC-ND 4.0), which permits the noncommercial replication and distribution of the article with the strict proviso that no changes or edits are made and the original work is properly cited (including links to both the formal publication through the relevant DOI and the license). See: https://creativecommons.org/licenses/by-nc-nd/4.0/.

\section{References}

1. Germain DP. Fabry disease. Orphanet J Rare Dis 2010;5:30.

2. Ramaswami U, Najafian B, Schieppati A, et al. Assessment of renal pathology and dysfunction in children with Fabry disease. Clin J Am Soc Nephrol 2010;5:365-70.

3. Waldek S, Patel MR, Banikazemi M, et al. Life expectancy and cause of death in males and females with Fabry disease: findings from the Fabry Registry. Genet Med 2009;11:790-6.

4. Germain DP, Nicholls K, Giugliani R, et al. Efficacy of the pharmacologic chaperone migalastat in a subset of male patients with the classic phenotype of Fabry disease and migalastat-amenable variants: data from the phase 3 randomized, multicenter, double-blind clinical trial and extension study. Genet Med 2019;21:1987-97.

5. Hughes DA, Nicholls K, Shankar SP, et al. Oral pharmacological chaperone migalastat compared with enzyme replacement therapy in Fabry disease: 18-month results from the randomised phase III ATTRACT study. J Med Genet 2017;54:288-96.

6. Feldt-Rasmussen U, Hughes D, Sunder-Plassmann G, et al. Long-term efficacy and safety of migalastat treatment in Fabry disease: 30-month results from the open-label extension of the randomized, phase 3 ATTRACT study. Mol Genet Metab 2020;131:219-28.

7. Simonetta I, Tuttolomondo A, Daidone $M$, et al. Biomarkers in Anderson-Fabry Disease. Int J Mol Sci 2020;21:8080.

8. Nowak A, Mechtler T, Kasper DC, et al. Correlation of Lyso-Gb3 levels in dried blood spots and sera from patients with classic and Later-Onset Fabry disease. Mol Genet Metab 2017;121:320-4.

9. Nowak A, Mechtler TP, Hornemann T, et al. Genotype, phenotype and disease severity reflected by serum LysoGb3 levels in patients with Fabry disease. Mol Genet Metab 2018;123:148-53.

10. Wilcox WR, Oliveira JP, Hopkin RJ, et al. Females with Fabry disease frequently have major organ involvement: lessons from the Fabry Registry. Mol Genet Metab 2008;93:112-28.

11. Tøndel C, Bostad L, Hirth A, et al. Renal biopsy findings 
in children and adolescents with Fabry disease and minimal albuminuria. Am J Kidney Dis 2008;51:767-76.

12. Waldek S, Feriozzi S. Fabry nephropathy: a review - how can we optimize the management of Fabry nephropathy? BMC Nephrol 2014;15:72.

13. Germain DP, Waldek S, Banikazemi M, et al. Sustained, long-term renal stabilization after 54 months of agalsidase beta therapy in patients with Fabry disease. J Am Soc Nephrol 2007;18:1547-57.

14. Mehta A, Ricci R, Widmer U, et al. Fabry disease defined: baseline clinical manifestations of 366 patients in the Fabry Outcome Survey. Eur J Clin Invest 2004;34:236-42.

15. Chen HC, Tsai JH, Lai YH, et al. Renal changes in heterozygous Fabry's disease--a family study. Am J Kidney Dis 1990;15:180-3.

16. Kleinert J, Dehout F, Schwarting A, et al. Prevalence of uncontrolled hypertension in patients with Fabry disease. Am J Hypertens 2006;19:782-7.

17. Ries M, Bettis KEB, Choyke P, et al. Parapelvic kidney cysts: a distinguishing feature with high prevalence in Fabry disease. Kidney Int 2004;66:978-82.

18. Pisani A, Petruzzelli Annicchiarico L, Pellegrino A, et al. Parapelvic cysts, a distinguishing feature of renal Fabry disease. Nephrol Dial Transplant 2018;33:318-23.

19. Grünfeld JP, Lidove O, Joly D, et al. Renal disease in Fabry patients. J Inherit Metab Dis 2001;24 Suppl 2:71-4; discussion 65 .

20. Branton MH, Schiffmann R, Sabnis SG, et al. Natural history of Fabry renal disease: influence of alphagalactosidase A activity and genetic mutations on clinical course. Medicine (Baltimore) 2002;81:122-38.

21. Tøndel C, Bostad L, Larsen KK, et al. Agalsidase benefits renal histology in young patients with Fabry disease. J Am Soc Nephrol 2013;24:137-48.

22. Mignani R, Feriozzi S, Schaefer RM, et al. Dialysis and transplantation in Fabry disease: indications for enzyme replacement therapy. Clin J Am Soc Nephrol 2010;5:379-85.

23. Cybulla M, Kurschat C, West M, et al. Kidney transplantation and enzyme replacement therapy in patients with Fabry disease. J Nephrol 2013;26:645-51.

24. Ojo A, Meier-Kriesche HU, Friedman G, et al. Excellent outcome of renal transplantation in patients with Fabry's disease. Transplantation 2000;69:2337-9.

25. Ersözlü S, Desnick RJ, Huynh-Do U, et al. Long-term Outcomes of Kidney Transplantation in Fabry Disease. Transplantation 2018;102:1924-33.

26. Shah T, Gill J, Malhotra N, et al. Kidney transplant outcomes in patients with Fabry disease. Transplantation 2009;87:280-5.

27. Desnick RJ, Wasserstein MP. Fabry disease: clinical features and recent advances in enzyme replacement therapy. Adv Nephrol Necker Hosp 2001;31:317-39.

28. Capelli I, Aiello V, Gasperoni L, et al. Kidney Transplant in Fabry Disease: A Revision of the Literature. Medicina (Kaunas) 2020;56:284.

29. Gantenbein H, Bruder E, Burger HR, et al. Recurrence of Fabry's disease in a renal allograft 14 years after transplantation. Nephrol Dial Transplant 1995;10:287-9.

30. Minami M, Mizuma E, Nakahara M, et al. A case of latent heterozygous Fabry disease in a female living kidney donor candidate. CEN Case Rep. 2021;10:30-4.

31. Colpart P, Félix S. Fabry Nephropathy. Arch Pathol Lab Med 2017;141:1127-31.

32. Eikrem Ø, Skrunes R, Tøndel C, et al. Pathomechanisms of renal Fabry disease. Cell Tissue Res 2017;369:53-62.

33. Fogo AB, Bostad L, Svarstad E, et al. Scoring system for renal pathology in Fabry disease: report of the International Study Group of Fabry Nephropathy (ISGFN). Nephrol Dial Transplant 2010;25:2168-77.

34. Kanai T, Yamagata T, Ito T, et al. Foot process effacement with normal urinalysis in classic fabry disease. JIMD Rep 2011;1:39-42.

35. Najafian B, Svarstad E, Bostad L, et al. Progressive podocyte injury and globotriaosylceramide (GL-3) accumulation in young patients with Fabry disease. Kidney Int 2011;79:663-70.

36. Najafian B, Tøndel C, Svarstad E, et al. Accumulation of Globotriaosylceramide in Podocytes in Fabry Nephropathy Is Associated with Progressive Podocyte Loss. J Am Soc Nephrol 2020;31:865-75.

37. Sperati CJ, Rosenberg AZ. Hydroxychloroquine-induced mimic of renal Fabry disease. Kidney Int 2018;94:634.

38. Sheth KJ, Roth DA, Adams MB. Early renal failure in Fabry's disease. Am J Kidney Dis 1983;2:651-4.

39. Slaats GG, Braun F, Hoehne M, et al. Urine-derived cells: a promising diagnostic tool in Fabry disease patients. Sci Rep 2018;8:11042.

40. Sanchez-Niño MD, Perez-Gomez MV, Valiño-Rivas L, et al. Podocyturia: why it may have added value in rare diseases. Clin Kidney J 2019;12:49-52.

41. Ortiz A, Oliveira JP, Waldek S, et al. Nephropathy in males and females with Fabry disease: cross-sectional description of patients before treatment with enzyme replacement therapy. Nephrol Dial Transplant 2008;23:1600-7.

42. Selvarajah M, Nicholls K, Hewitson TD, et al. Targeted 
urine microscopy in Anderson-Fabry disease: a cheap, sensitive and specific diagnostic technique. Nephrol Dial Transplant 2011;26:3195-202.

43. Nakamichi T, Miyazaki M, Nakayama K, et al. Fabry's disease discovered with chance urinary mulberry cells: a case report. Clin Kidney J 2018;12:49-52.

44. Trimarchi H, Canzonieri R, Schiel A, et al. Podocyturia is significantly elevated in untreated vs treated Fabry adult patients. J Nephrol 2016;29:791-7.

45. Schiffmann R, Waldek S, Benigni A,et al. Biomarkers of Fabry disease nephropathy. Clin J Am Soc Nephrol 2010;5:360-4.

46. Feriozzi S, Germain DP, Di Vito R, et al. Cystatin C as a marker of early changes of renal function in Fabry nephropathy. J Nephrol 2007;20:437-43.

47. Hilz MJ. Evaluation of peripheral and autonomic nerve function in Fabry disease. Acta Paediatr Suppl 2002;91:38-42.

48. Feriozzi S, Torras J, Cybulla M, et al. The Effectiveness of Long-Term Agalsidase Alfa Therapy in the Treatment of Fabry Nephropathy. Clin J Am Soc Nephrol 2012;7:60-9.

Cite this article as: Kurschat CE. Fabry disease-what cardiologists can learn from the nephrologist: a narrative review. Cardiovasc Diagn Ther 2021;11(2):672-682. doi: 10.21037/cdt20-981
49. Mehta A, Beck M, Elliott P, et al. Enzyme replacement therapy with agalsidase alfa in patients with Fabry's disease: an analysis of registry data. Lancet 2009;374:1986-96.

50. Beck M, Hughes D, Kampmann C, et al. Long-term effectiveness of agalsidase alfa enzyme replacement in Fabry disease: A Fabry Outcome Survey analysis. Mol Genet Metab Rep 2015;3:21-7.

51. Germain DP, Charrow J, Desnick RJ, et al. Ten-year outcome of enzyme replacement therapy with agalsidase beta in patients with Fabry disease. J Med Genet 2015;52:353-8.

52. Banikazemi M, Bultas J, Waldek S, et al. Agalsidase-beta therapy for advanced Fabry disease: a randomized trial. Ann Intern Med 2007;146:77-86.

53. Breunig F, Weidemann F, Strotmann J, et al. Clinical benefit of enzyme replacement therapy in Fabry disease. Kidney Int 2006;69:1216-21.

54. Lenders M, Brand E. Effects of Enzyme Replacement Therapy and Antidrug Antibodies in Patients with Fabry Disease. J Am Soc Nephrol 2018;29:2265-78. 